\title{
Perfil de internações clínicas em hospital secundário do interior do Rio Grande do Sul. O caso do Hospital de Caridade de São Jerônimo.
}

\author{
Gisele B. Piccoli ${ }^{1}$; Paula L. Dutra²; Alexandra Daniel'² Cezar V. W. \\ Riche $^{2}$; José Augusto S. Pellegrini³; André Wajner ${ }^{3}$
}

\section{Resumo}

Introdução: Os dados sobre perfil de pacientes clínicos que internam em hospitais secundários são raros no Brasil. Porém, o conhecimento a respeito desta população tem grande importância no estabelecimento das políticas públicas de saúde e na gestão de leitos. Em pesquisa de 2014, foi realizada primeira caracterização da população de pacientes clínicos internados em hospital secundário que obteve dados de 1149 internações em 8 meses.

Objetivo: Caracterizar os pacientes clínicos SUS internados em hospital secundário da região carbonífera do Rio Grande do Sul, na cidade de São Jerônimo.

Método: Realizado estudo coorte retrospectivo em banco de dados do Serviço de Medicina Hospitalar. A coleta de dados é realizada prospectivamente em banco de dados do Excel®. Neste estudo foi utilizada estatística descritiva.

Resultados: No período de março de 2015 a fevereiro de 2016, houve 1544 internações. A média de idade dos pacientes foi de 65,7 anos, com predominância de pacientes masculinos, 53\%. Os pacientes eram provenientes da própria cidade em $34 \%$ das vezes. O tempo médio de internação foi de 7,7 dias (desvio padrão 5,7), mediana de 6,7 dias com intervalo interquartil (IQ 25-75) de 3,9-9,7. As principais causas de internação foram: afecções pulmonares $18 \%$, infecções $11 \%$, afecções cardíacas $8 \%$, afecções gastrointestinais $6 \%$ e afecções neurológicas 3\%. A mortalidade geral foi de $10 \%$ e a taxa de re-internação em 30 dias de $9,5 \%$.

Conclusão: Os dados apresentam o perfil das internações de pacientes clínicos de um hospital secundário do interior do Rio Grande do Sul. Contribuem para a construção do conhecimento de suas características e 
planejamento do perfil de equipes assistenciais necessárias. Do ponto de vista médico os autores acreditam que equipes dotadas de profissionais com especialização em clínica médica são adequadas para instituições semelhantes.

Palavras Chave: pacientes clínicos, medicina hospitalar, hospital secundário. 